\title{
GESTÃO DA INFORMAÇÃO E O SISTEMA SMART GRID: UM ESTUDO DE CASO DA UFRN
}

ARAÚJO, Arthur Nóbrega B. de. Graduando em Engenharia de Produção pela Universidade Federal do Rio Grande do Norte - UFRN. Telefone: (84) 8802-3966. E-mail: araujo.arthur@ymail.com.

QUEIROZ, Jamerson Viegas. Dr. em engenharia de Produção, professor do programa de Pós-Graduação em Engenharia de Produção da Universidade Federal do Rio Grande do Norte - UFRN. Telefone: (84) 3642-

1839. E-mail: jvqjamerson@yahoo.com.br.

FERNANDES, Helton Tadeu P.; GOMES, João Paulo Bernardo da S.; MORAIS, Adriano Varella de.

Graduandos em Engenharia de Produção pela Universidade Federal do Rio Grande do Norte - UFRN.

\begin{abstract}
RESUMo
A eficiência energética e a confiabilidade na distribuição de energia elétrica são tendências de foco nesse tipo de serviço. O presente trabalho defende que uso de tecnologia de informação e automação em redes elétricas a fim de torná-las inteligentes (Smart Grid) melhora o processo de tomada de decisão. A metodologia adotada foi uma análise conceitual a partir da hierarquia de valor dos sistemas de informação, utilizando um estudo comparativo desse tipo de tecnologia com a usada na Universidade Federal do Rio Grande do Norte. Conclui que a automação de processos aumenta a eficiência do processo, bem como a eficiência da tomada decisão, mas exige uma estratégia voltada para a gestão do conhecimento.
\end{abstract}

Palavras-chave: Sistemas de informação; Tecnologia de automação; Processo decisório; Redes elétricas inteligentes.

\section{Abstract}

The energetic efficiency and the reliability in the electricity distribution are tendencies in thiskind of service. The present essay advocates that the utilization of information and automation technology in electric grids, in order to make them intelligent (Smart Grid), improves the decision-making process. It was used a conceptual analysis about the information system's value hierarchy in the methodology, making a comparative study of this kind of technology with the one used at the Universidade Federal do Rio Grande do Norte. We concluded that the automation in processes improves its efficiency, as well as the efficiency in the decision-making process, but it requires a strategy that is focused in knowledge management.

KEYWORDS: Information systems; Automation technology; Decision process; Intelligent electric grids. 


\section{INTRODUÇÃo}

O setor de energia elétrica é de importância estratégica para o bem-estar da indústria de um país, pois faz parte da infraestrutura básica para $o$ funcionamento desta. Dessa forma, pode-se inferir que a qualidade nesse setor é fundamental, assim como afirma Chowdhury et al. (2003).

Em virtude de ser uma atividade econômica altamente regulamentada, de utilidade pública e, pela sua natureza, com características de monopólio, é de suma importância a verificação periódica da qualidade do serviço de fornecimento de energia elétrica prestado aos consumidores pelas concessionárias (SILVESTRE et al., 2010, p. 98).

O foco da qualidade na distribuição de eletricidade, em relação ao consumidor, está diretamente associado à confiabilidade, como defende Billinton et al. (1997). Além disso, Cassula et al. (2003) afirmam que a confiabilidade é de suma importância na transmissão de energia elétrica, pois esse serviço exige altos investimentos e tem impacto direto no meio ambiente e na sociedade.

Assim, é interessante buscar alternativas que minimizem as falhas que causem quedas de energia, como o próprio crescimento da demanda, que sobrecarrega a rede e interrompe o fornecimento. Para tal, Madani e King (2011) abordam a importância do uso da tecnologia de informação e monitoramento para a redução de falhas em redes elétricas. Em âmbito mais geral, Queiroz e Vasconcelos (2005) defendem que a implantação de tecnologia de informação e automação aumenta a eficiência nas organizações, contanto que seja elaborada uma estratégia voltada para a adaptação do trabalhador a essa tecnologia.

O presente trabalho busca avaliar como a estruturação de uma Smart Grid (rede inteligente) na Universidade Federal do Rio Grande do Norte pode afetar o processo de tomada de decisão em relação aos problemas de distribuição de energia elétrica, uma vez que essa organização passa por problemas de frequentes quedas de energia, o que caracteriza baixa confiabilidade da rede.

A organização do presente estudo inicia-se com essa abordagem introdutória, seguida pela seção 2, que discute a revisão da literatura; o capítulo 3 apresenta a metodologia; na seção 4 é ressaltado o estudo de caso da UFRN; a seção 5 apresenta os resultados encontrados; e, por último, a seção 6 trata das considerações finais.

\section{REFERENCIAL TEÓRICO}

\section{Eficiência energética e redes elétricas}

Eficiência é a relação entre o resultado alcançado e os recursos que foram utilizados na operação. A eficiência energética é o principal foco de uma rede elétrica inteligente, que através do autogerenciamento da rede busca realizar o consumo da energia de forma ideal, reduzindo perdas desde a geração até a distribuição para o consumidor final. Um sistema inteligente se torna mais eficiente pelo fato de tomar decisões em tempo real, utilizando a energia de modo racional.

[...] o uso intenso das fontes de energia conhecidas e seus impactos adversos sobre o ambiente, problemas diretamente relacionados aos sistemas de energia elétrica, também aparecem sob o pálio do desenvolvimento sustentável. Por esses motivos, o impacto ambiental é um fator de relevância crescente e de importância nas condições atuais de operação e de desenvolvimento desses sistemas e, indiscutivelmente, deve ter um efeito mais intenso sobre a indústria no futuro (GÓMEZEXPÓSITO, 2011).

Há também a necessidade de garantir a eficiência operacional do sistema elétrico em todas as suas etapas, a partir do gerenciamento correto da rede de energia elétrica. As perdas devem ser diminuídas e os impactos ambientais, reduzidos. Com isso, as empresas se tornam mais competitivas em razão do meio ambiente, mas claramente em função da eficiência, da redução de custos e despesas causadas pela dificuldade de 
produzir de forma correta.

A maioria dos equipamentos e processos utilizados nos dias de hoje nos setores de transporte, industrial e residencial foi desenvolvida numa época de energia abundante e barata e quando as preocupações ambientais ou não existiam ou eram pouco compreendidas. Estes são os motivos pelos quais haja tantas oportunidades para melhorias na economia de energia, seja para aumentar a competitividade das empresas, seja para melhorar a imagem pública de indústrias que deixaram de ser poluentes (GOLDEMBERG, 2000, p. 93).

\section{Sistemas de informação e automação}

O cerne da administração de organizações está na tomada de decisão, e os sistemas de informação são responsáveis por dar suporte aos gerentes para tal. Guimarães e Évora (2004) afirmam que "[...] a informação é um recurso primordial para tomada de decisão e que, quanto mais estruturado for este processo, [...] mais indicado se faz o uso de sistemas de informação".

Moresi (2000) aborda a cadeia de valor de um sistema de informação, que por sua vez é sustentado por algum tipo de tecnologia de informação. Essa cadeia é mostrada na Figura 1.

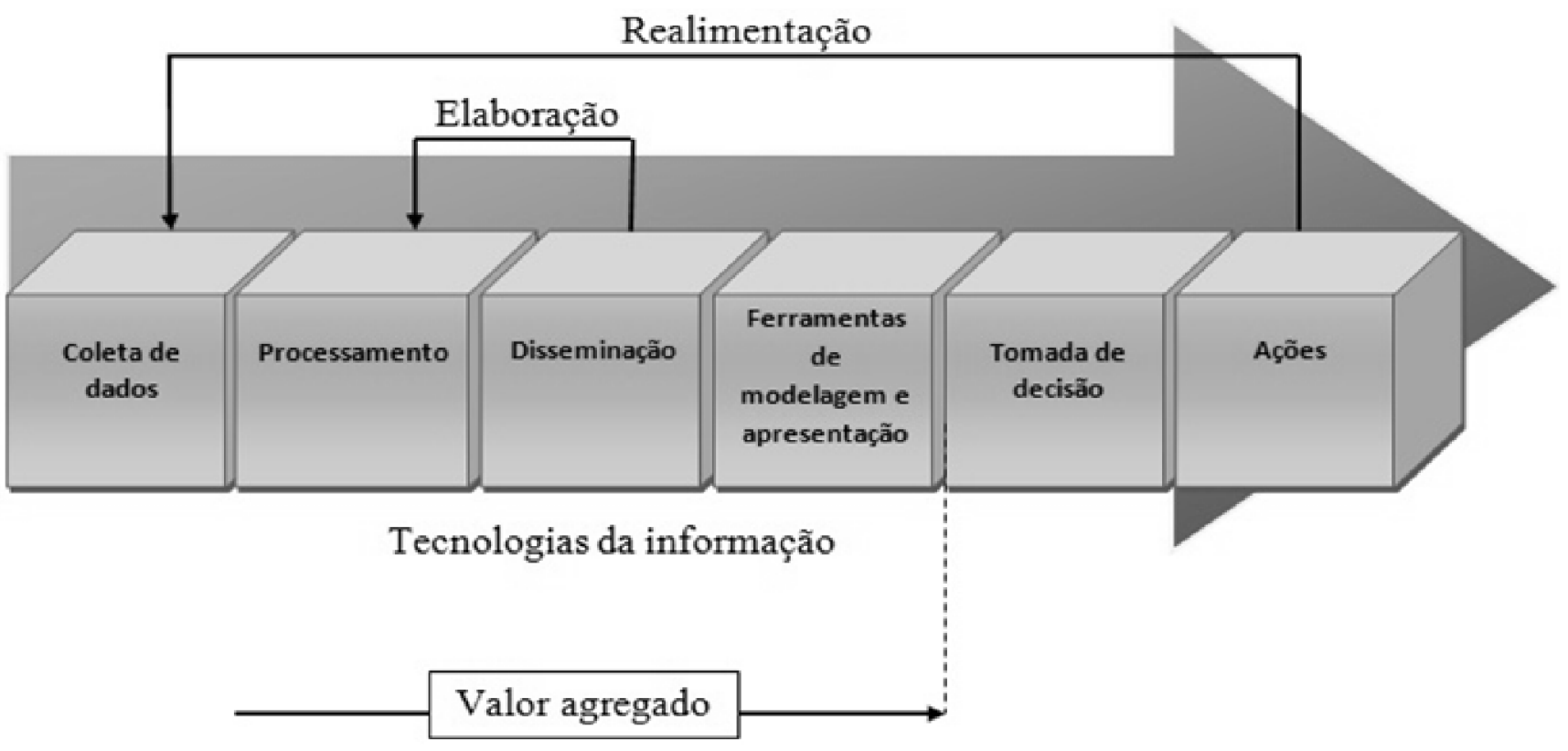

Figura 1 - Cadeia de valor dos sistemas de informação.

Fonte: Adaptado de Moresi (2000).

Como mostrado, a tecnologia de informação tem seu valor acrescido de acordo com seu desempenho nessa cadeia de valor. Se um sistema apenas coleta dados, seu valor será inferior em relação ao que processa.

Slack et al. (2009) dividem os sistemas de informação em três tipos diferentes: o de suporte à decisão, que coleta, processa e dissemina dados a fim de informar o gerente e apoiar sua decisão; o de informação gerencial, que agrega ainda mais valor, chegando a usar ferramentas de modelagem e apresentação dos dados; e ainda os sistemas especialistas, que tomam decisões para resolver problemas limitados. 
A partir daí, é possível falar em automação flexível, que, de acordo com Zukin e Dalcol (2001), é uma integração entre tecnologia de informação - TI e processo de manufatura automatizado, através de computador. ATI usada na automação é justamente a de sistemas especializados, chegando a tomar decisões e inclusive agir, ou seja, esse tipo de tecnologia está no topo da cadeia de valor dos sistemas de informação.

A automação aumenta a flexibilidade do processo produtivo, pois reduz a burocracia na tomada de decisão, uma vez que ela passa a acontecer em nível operacional, e não tático. Mesmo assim, é importante ressaltar que esse tipo de tecnologia não retira a necessidade de pessoas no processo decisivo, já que o sistema precisa ser gerenciado e suas decisões são limitadas a processos repetitivos e/ou simples.

Sordi e Medeiros Júnior (2006) defendem que o uso de uma única plataforma integradora é mais interessante para gerenciar as informações, pois isso aumenta a confiabilidade da informação, o número de possíveis análises com a mesma informação, e ainda facilita o processo de automação.

\section{A rede elétrica inteligente}

A tradução do termo Smart Grid já transmite a essência do seu significado: rede inteligente. Esta rede é a aplicação de tecnologia de informação para o sistema elétrico de potência, integrada aos sistemas de comunicação e infraestrutura de rede automatizada.

Esse conjunto tecnológico seria o responsável por significativos ganhos de eficiência energética, por permitir automação e operação remota do sistema, por melhorar a fiscalização e monitoramento das condições de rede e qualidade de energia, por incrementar a capacidade de tomada de decisões nas diferentes fases do setor, por viabilizar tecnicamente ao consumo programado, inteligente, de energia, dentre outros (RIBEIRO, 2011, p. 3).

Para alcançar esses resultados, a Smart Grid conta com três pontos principais: o sensoriamento, a telecomunicação e o processamento.

O sensoriamento exerce o papel de captação das informações sobre a operação e o desempenho da rede, apresentando, por meio dos medidores inteligentes (uma das ferramentas do sistema), parâmetros como tensão e corrente, e após uma análise fornece as informações.

A telecomunicação exerce a função de transmitir as informações coletadas da rede. Esta transmissão pode ocorrer de duas maneiras: com a Power Line Communications, tecnologia que usa a própria rede elétrica para transmitir os dados, ou com tecnologias de transmissão de dados desvencilhadas da rede de energia elétrica (GSM, GPRS, UMTS, SMS, etc). O processamento, último ponto, exerce a função de interpretar as informações em trânsito e tomar decisões de forma independente, entre outras coisas.

A Smart Grid funciona a partir de sensores que, quando detectam informações significativas, comunicam os dados de volta para um sistema analítico central. Este sistema é representado, geralmente, por um software que irá analisar os dados e determinar o que há de errado na rede e o que deve ser feito para melhorar o desempenho dela.

Essa tecnologia proporciona três grandes benefícios. O primeiro deles é a eficiência energética. A confiabilidade aparece como segundo benefício, pois a rede tem a capacidade de detectar quando os ativos estão funcionando de maneira irregular (risco de falha, desempenho em declínio, etc.) e identificálos para a concessionária repará-los antes de uma interrupção real.

A tecnologia da Smart Grid permite localizar a falha com precisão, respondendo de maneira mais rápida ao funcionamento incorreto e minimizando o impacto das falhas aos clientes, isolando uma região menor e fazendo com que menos clientes sejam afetados.

Por último, há a integração de ponta que varia desde o sistema de gestão do cliente em casa, através da leitura dos medidores inteligentes, até o controle e acompanhamento da integração de outras fontes, como as placas solares. Existe também o abastecimento de veículos elétricos que exigem a interação com a rede 
para ser bem-sucedido.

\section{Metodologia}

A princípio, foirealizada uma pesquisa bibliográfica descritiva sobre a rede Smart Grid, e percebeu-se que o foco no desenvolvimento desse tipo de sistema não está na redução de custos, e sim na simplificação da tomada de decisão e no aumento da qualidade da distribuição de energia no que diz respeito à confiabilidade da rede.

Além de pesquisa bibliográfica, foram realizadas discussões com o intuito de definir os objetivos do trabalho, o que foi feito a partir das necessidades mais urgentes observadas, no caso, a resolução do problema da baixa confiabilidade da rede.

Com um objetivo definido, a pesquisa de referencial teórico começou com os sistemas de informação e automação e seus impactos no processo decisório, além das questões ambientais relacionadas à eficiência energética, fatores diretamente ligados às quedas de energia. Em seguida, foi realizado um estudo mais aprofundado da instituição, avaliando como é feito o gerenciamento da rede e quais seus pontos fortes, fracos, oportunidades e ameaças.

A partir daí, foi montada uma matriz SWOT, com o intuito de averiguar a viabilidade estratégica da instalação desse sistema. Na matriz, foram usados índices de correlação entre os aspectos externos (oportunidades ou ameaças) e os aspectos internos (forças ou fraquezas). Caso a relação fosse grande, usou-se o índice 9, média, 5, pequena, 1 , e inexistente, 0 .

Os resultados foram usados para explorar as formas de influência que um sistema de informação e automação tem na tomada de decisão, especificando o caso da UFRN, bem como levantar as vantagens no gerenciamento de energia que esse tipo de tecnologia pode trazer.

\section{ESTUDO DE CASO - UMA REDE INTELIGENTE NA UFRN Situação energética da UFRN}

Como já mencionado, a proposta do trabalho é estudar a viabilidade da implantação de uma Smart Grid na UFRN, logo, ela é o cliente do negócio. Foi aplicado um questionário na Superintendência de Infraestrutura acerca do contexto energético na universidade e, a partir dele, o cliente foi caracterizado.

O crescimento médio, nos últimos cinco anos, do consumo de energia elétrica na UFRN é de cerca de $25 \%$ por ano. Existem duas redes que abastecem a instituição, as subestações de Neópolis e de Lagoa Nova. Os dois contratos de energia (um para cada rede) totalizam seis mega Watts de potência elétrica.

Segundo a própria Superintendência de Infraestrutura, as principais causas para as frequentes quedas de energia em alguns setores da universidade são falhas da concessionária e sobrecargas da rede, gerada por um aumento significativo da demanda energética.

A rede elétrica na UFRN não á automatizada, havendo apenas um sistema de monitoramento. Assim, quando um circuito entra em sobrecarga, outro que esteja suboperado não pode abastecê-lo. Por causa disso, estão sendo realizados investimentos em infraestrutura energética, como a construção de subestações próprias da UFRN e expansões das linhas de distribuição.

Existem equipes de manutenção da rede elétrica, que operam caso haja falhas. Por exemplo, em caso de sobrecarga, eles são responsáveis por desligar circuitos de menor prioridade a fim de reduzir a demanda por eletricidade e a rede voltar a transmitir. Um sistema de automatização da rede permitiria realizar esse tipo de ação preventivamente e sem ter que acionar a equipe de manutenção.

\section{O sistema atual}

O sistema de gerenciamento de energia elétrica utilizado pela Universidade Federal do Rio Grande do Norte possibilita o acompanhamento do consumo de energia elétrica objetivando o melhoramento da gestão energética na organização.

Ele tem como característica funcional o fornecimento de detalhes das despesas mensais com o consumo energético, para que se tenham informações sobre comportamento (consumo ao logo do tempo), possibilitando o planejamento de ações que possam 
ser aplicadas para minimizar um possível desperdício de energia, além de projetar a oferta para demandas imediatas que ocorram na comunidade acadêmica.

$\mathrm{O}$ atual sistema também fornece subsídios para identificação de algumas perdas motivadas por ações passadas e atuais, como:

i. Cultura do desperdício de energia;

ii. Penalidades por contratos de energia mal elaborados, devido a demandas mal dimensionadas;

iii. Descontrole nas medições do consumo;

iv. Dimensionamento inadequado de fontes consumidoras de energia, tais como a iluminação além da real necessidade, sobre dimensionamento de motores, entre outros;

v. Sobrecarga nos transformadores e cabos de energia;

vi. Inexatidão das contas de energia;

vii. Inadequações nas chaves de rateio de custos.

Esse sistema também permite o monitoramento de fluxos de energia em utilidades em tempo real, em que pode ser feito o acompanhamento da medição de energia, medição de utilidades, supervisão da demanda e do consumo e acionamento de alarmes de problemas na rede, como também a verificação do fator de potência, rateio de custo, qualidade da energia e simulações no sistema.

Portanto, o sistema atual é de suporte de informação gerencial, o qual coleta dados sobre o comportamento da rede elétrica para subsidiar a geração de relatórios analíticos e vários gráficos. Só então poderá ser realizada alguma ação pelos técnicos de manutenção para a correção do problema ou minimização de seus efeitos.

\section{Resultados ENCONTRADOS}

\section{Comparações entre uma Smart Grid e a rede} atual

Ao analisar o sistema da Smart Grid, foram identificadas suas funcionalidades, as quais possibilitam: a distribuição de pacotes de energia para cada unidade consumidora; o gerenciamento do consumo de energia em tempo real, diminuído o lead time; o fornecimento, em tempo real, do gasto de energia em reais da unidade consumidora; a automação do sistema possibilita realização de operações de forma preventiva, como desligamento de equipamentos que estejam aumentando o consumo na ponta; evitar o sobredimensionamento da infraestrutura da rede elétrica para alguns momentos de pico (em torno de $10 \%$ do dia); preparar a UFRN à realidade futura da distribuição energia pelas concessionárias.

Na investigação sobre o sistema de monitoramento de distribuição energética da UFRN, verificou-se que ele possibilita: alertar sobre ultrapassagem do consumo preestabelecido; monitoramento da distribuição de energia; fornecimento do consumo energético, em tempo real; fornecimento de dados para que sejam tomadas as decisões pelo departamento.

Assim, percebe-se que a principal diferença entre os sistemas é que a tecnologia da Smart Grid é um sistema especialista, possibilitando a automação flexível, enquanto o sistema atual é de informação gerencial. Dessa forma, a rede inteligente reduz a burocracia na tomada de decisão, que passa a ser feita no âmbito operacional em vez do tático, o que torna as ações realizadas preventivas. O sistema atual dá suporte aos gestores para que eles tomem a decisão, mas sempre de forma corretiva.

A rede inteligente ainda permite alocar potência elétrica em circuitos que têm maiores demandas, reduzindo o fornecimento para os que têm menores demandas, o que reduz o desperdício no fluxo de eletricidade. Outro fato é a possibilidade de cortar circuitos de menor prioridade para evitar quedas de energia em circuitos prioritários, evitando assim as quedas de energia nas ocasiões de sobrecarga. Dessa forma, se torna desnecessário sub-redimensionar a infraestrutura elétrica para picos de demanda isolados, reduzindo os investimentos em subestações de distribuição. 


\section{MATRIZ SWOT}

\begin{tabular}{|c|c|c|c|c|c|c|c|c|c|c|}
\hline \multirow{2}{*}{\multicolumn{2}{|c|}{ AMBIENTE }} & \multicolumn{3}{|c|}{ PONTOS FRACOS } & \multirow[b]{2}{*}{ 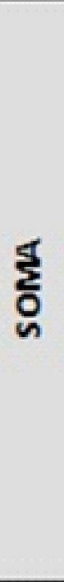 } & \multicolumn{4}{|c|}{ PONTOS FORTES } & \multirow[b]{2}{*}{$\sum_{\substack{5\\
}}^{5}$} \\
\hline & & 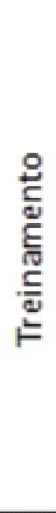 & 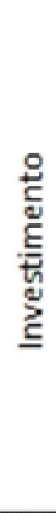 & 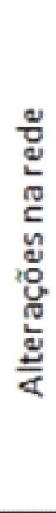 & & 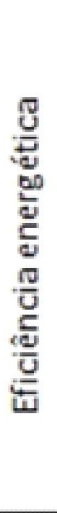 & 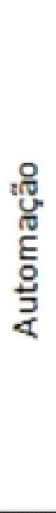 & 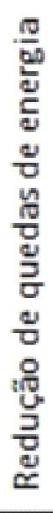 & 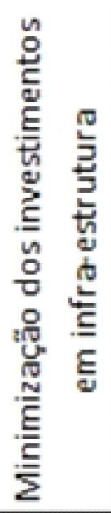 & \\
\hline \multirow{2}{*}{ 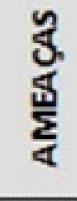 } & Burocracia & 5 & 9 & 9 & 23 & 0 & 9 & 1 & 9 & 19 \\
\hline & $\begin{array}{l}\text { Resistência a } \\
\text { mudanças }\end{array}$ & 9 & 0 & 5 & 14 & 0 & 9 & 0 & 0 & 9 \\
\hline \multicolumn{2}{|r|}{ SOMA } & 14 & 9 & 14 & 37 & 0 & 18 & 1 & 9 & 28 \\
\hline \multirow{3}{*}{ 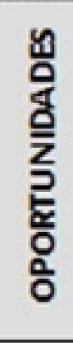 } & $\begin{array}{l}\text { Mudança da infra- } \\
\text { estrutura elétrica }\end{array}$ & 0 & 9 & 9 & 18 & 9 & 5 & 9 & 9 & 32 \\
\hline & $\begin{array}{c}\text { Potencial intelectual } \\
\text { na universidade }\end{array}$ & 9 & 0 & 1 & 10 & 0 & 9 & 0 & 0 & 9 \\
\hline & $\begin{array}{c}\text { Existência da estrutura } \\
\text { gerencial }\end{array}$ & 9 & 9 & 1 & 19 & 0 & 5 & 0 & 5 & 10 \\
\hline \multicolumn{2}{|r|}{ SOMA } & 18 & 18 & 11 & 47 & 9 & 19 & 9 & 14 & 51 \\
\hline
\end{tabular}

Figura 2 - Matriz SWOT de uma Smart Grid para a UFRN.

Fonte: Elaboração própria.

De acordo com o resultado da matriz, a postura estratégica a ser adotada é a de desenvolvimento, o que prova a viabilidade estratégica do projeto. Isso se deve ao fato de a maior pontuação na matriz ter sido no quadrante que analisa pontos fortes e oportunidades, $\mathrm{o}$ que indica que a UFRN possui real necessidade de um sistema desse tipo (oportunidades) e o sistema realmente atende aos requisitos (pontos fortes).

A postura estratégica de desenvolvimento prevê $\mathrm{o}$ investimento em inovação, uma vez que tanto o ambiente interno como o externo estão favoráveis ao crescimento do negócio. Assim, o projeto de instalação de uma Smart Grid vem a calhar, pois elimina problemas que a universidade enfrenta atualmente e provê sustentação para um crescimento futuro.

Como mostra a matriz, alguns aspectos internos que se destacam nos resultados são treinamento e automação. Isso demonstra a importância do gerenciamento do sistema após sua instalação, sendo imprescindível treinar os funcionários, devido à automação da rede, que altera as funções e atividades da gerência, que deixa de ter papel de corrigir problemas e passa a controlar o funcionamento do software. 
Em relação aos aspectos externos, verifica-se que o a burocracia dentro das instituições públicas, bem como a existência de uma estrutura gerencial, influenciam fortemente nos resultados. Assim, é necessário que a Superintendência de Infraestrutura participe da instalação do sistema.

\section{Smart Grid e suas consequências na tomada de decisão}

Amaral e Sousa (2011) afirmam que as decisões podem ser tratadas como algo científico e racional, enfatizando-se as análises e as relações de causa e efeito, com o intuito de antecipar ações e decidir de forma mais eficaz e eficiente.

A rede inteligente possibilita o downsizing, que nada mais é do que antecipação da tomada de decisão, que deixa de ser feita no âmbito estratégico ou tático, e passa a acontecer no operacional. Assim, o problema das quedas de energia seria reduzido em razão da característica proativa da Smart Grid.

Como defende Li et al. (2010), um dos principais benefícios da Smart Grid é a customização. Logo, a tomada de decisão relacionada às redes elétricas será direcionada aos indicadores que vão predeterminar as ações que o sistema realizará, bem como os índices de desempenho a serem usados para avaliar o próprio sistema.

Segundo Passos e Silva Filho (1994), definir indicadores em sistemas de informação e automação é algo complexo devido à dimensão e complexidade desses tipos de sistemas. Logo, haverá necessidade em treinar e especializar os agentes decisórios na gestão elétrica da organização.

De acordo com Ferreira (2008) apud Tomaselli (2010), decidir é o "ato que se apoia nos passos antecedentes da percepção e avaliação das condições oferecidas, constitui a essência dos atos humanos, ao reunir a capacidade de captar informações, analisálas e ponderar sobre elas, abrindo caminho, assim, para a função especial do pensar, que seguido pelo agir, pode criar e transformar".

A citação acima argumenta a necessidade de treinamento gerencial e desenvolvimento de uma política voltada para a gestão do conhecimento na gestão de uma Smart Grid. Essa é uma estratégia a ser priorizada no processo de implantação do sistema.

\section{Conclusões}

A importância da gestão da informação em organizações é ascendente com o passar do tempo, pois aumenta a eficiência na tomada de decisão e facilita o trabalho dos gerentes. Dessa forma, as organizações se tornam mais competitivas e aumentam seus reflexos às variações de mercado.

O uso de tecnologia de automação aumenta a eficiência dos processos produtivos, uma vez que possibilitam o downsizing. Esse aumento de eficiência possui consequências nos custos da empresa, na produtividade, na necessidade de investimentos e nos impactos ambientais gerados, que normalmente são reduzidos.

O uso de uma Smart Grid em redes elétricas permite esse aumento de eficiência, reduzindo os impactos ambientais na distribuição de eletricidade, devido ao equilíbrio entre demanda e oferta que é proporcionado, além do aumento da qualidade e confiabilidade da rede.

Desenvolver esse sistema é uma forma de produzir conhecimento, além de reduzir o problema das quedas de energia existentes na UFRN, a partir do controle dos circuitos pelo próprio sistema. Logo, conclui-se que a implantação do sistema apresenta vantagens para o funcionamento da universidade como um todo.

Para pesquisas futuras, é interessante definir quais indicadores usar para determinar as ações do sistema, além de determinar que índices de desempenho são melhores para medir o funcionamento do sistema.

\section{REFERÊNCIAS}

AMARAL, S.A.; SOUSA, A. J. F. P. Qualidade da informação e intuição na tomada de decisão organizacional. Perspectivas em Ciência da Informação, v. 16, n. 1, p. 133-146, 2011.

BILLINTON, R.; SALVADERI, L.; MCCALLEY, 
J. D.; CHAO, H.; SEITZ, T.; ALLAN, R. N.; ODOM, J.; FALLON, C. Reliability issues in today's electric power utility environment. IEEE Transactions on Power Systems, v. 12, n. 4, p. 1708-1714, 1997.

CASSULA, A. M; SILVA, A. M. L.; MANSO, L. A. F.; BILLINTON, R. Avaliação da confiabilidade em sistemas de distribuição considerando falhas de geração e transmissão. Revista Controle \& Automação, v. 14, n. 3, p. 262-271, 2003.

CHOWDHURY, A. A.; AGARWAL, S. K.; KOVAL, D. O. Reliability modeling of distributed generation in conventional distribution systems planning and analysis. IEEE Transactions on Industry Applications, v. 39, n. 5, p. 14931498, 2003.

FERREIRA, V. R. M. Psicologia econômica: estudo do comportamento econômico e da tomada de decisão. São Paulo: Elsevier, 2008.

GOLDEMBERG, José. Pesquisa e desenvolvimento na área de energia. São Paulo em Perspectiva, v. 14, n. 3, p. 91-97, 2000.

GÓMEZ-EXPÓSITO, Antonio. Sistemas de energia elétrica - análise e operação. Rio de Janeiro: LTC, 2011.

GUIMARÃES, E. M. P.; ÉVORA, Y. D. M. Sistema de informação: instrumento para tomada de decisão no exercício da gerência. Ciência da Informação, v. 33, n. 1, p. 72-80, 2004.

LI, F.; QIAO, W.; SUN, H.; WAN, H.; WANG, J.; XIA, Y.; XU, Z.; ZHANG, P. Smart transmission grid: vision and framework. IEEE Transactions On Smart Grid, v. 1, n. 2, p. 168-177, 2010.

MADANI, Vahid; KING, Roger L. Strategies and roadmaps to meet grid challenges for safety and reliability. In: ANDERS, George; VACCARO, Alfredo. Innovations in power systems reliability. Londres: Springer, 2011.

MORESI, E. A. D. Delineando o valor do sistema de informação de uma organização. Ciência da Informação, v. 29, n. 1, p. 14-24, 2000.

PASSOS, C. A. S.; SILVA FILHO, O. S. Modelos analíticos para avaliação de sistemas flexíveis de manufatura. Gestão \& Produção, v. 1, n. 3, p. 290-304, 1994.

QUEIROZ, A. C. S.; VASCONCELOS, F. C. Organizações, confiabilidade e tecnologia. Revista de Administração de Empresas, v. 45, n. 3, p. 40-51, 2005.

RIBEIRO, C. L. Aspectos institucionais para o Smart Grid no Brasil: Riscos, oportunidades e desafios regulatórios. Núcleo de direito setorial e regulatório. Brasília: UnB, 2011.

SILVESTRE, B. S.; HALL, J.; MATOS, S.; FIGUEIRA, L. A. P. A. Privatização: bom ou ruim? Lições do setor de distribuição de energia elétrica do nordeste brasileiro. Revista deAdministração de Empresas, v. 50, n. 1, p. 94-111, 2010.

SLACK, Nigel; CHAMBERS, Stuart; JOHNSTON, Robert. Administração da produção. 3. ed. São Paulo: Atlas, 2009.

SORDI, J. O.; MEDEIROS JÚNIOR , G. Abordagem sistêmica para integração entre sistemas de informação e sua importância à gestão da operação: análise do caso GVT. Gestão \& Produção, v. 13, n. 1, p. 105-116, 2006.

TOMASELLI, Tatiana Renaux. AACP e a tomada de decisão em investimentos. Revista de Psicologia, v. 22, n. 3, p. 525-542, 2010. 
ARAÚJO et al.

ZUKIN, Marcio; DALCOL, Paulo R. T. Um estudo e flexibilidade de manufatura. Revista Produção, v. empírico sobre a correlação entre automação flexível 10, n. 2, p. 21-30, 2001.

RECEBIDO EM 13/2/2012

ACEITO EM 10/5/2012 